\title{
Informing immersive learning design research and practice from the epistemology of the Santiago School of Cognition
}

\author{
Keywords \\ Creative practice, Human experience, Immersive design, Santiago School of Cognition, User-centred design
}

The Santiago School of Cognition postulates that the process of intelligent cognition in any living system is a result of its ongoing process of adaptation to its medium. In other words, the very process of life in living systems is a process of cognition. It also establishes that human experience and cognition is embodied and enacted with the environment, through a continuous process of active perception and sense-making of the world. Coming from systems biology and founded on the concept of autopoiesis, literally meaning self-making, defining living systems as those that can reproduce and self-maintain themselves by creating their own parts, the Santiago school essentially offers an alternative epistemology for the understanding of human experience phenomena with digital tools and environments. It also provides a framework for the creation, design, development, implementation and use of digital affordances (possibilities offered by digital technology) in education and beyond. Informing immersive learning design research and practice from the epistemology of the Santiago school also helps exploring and navigating digital innovation and the emergence of new technologies and modes of user experience design and practice. Under the premise that the nature of the world we live in is complex, interconnected, unpredictable and ever-changing, and that human experience is subjective, ecosomaesthetic, symbolic and felt with the world, traditional western design concepts such as 'one solution fits all' or even the notion of 'user experience (UX) design' become problematic. Autopoiesis, cognition and enaction at the basis of human lived experience are some of the fundamental concepts and principles coming from the epistemology of the Santiago school that can inform and guide user-centred design and creative making practice in real and virtual worlds. Embedding properties found in living systems within creative solutions, or designing for users 'to become with the world' in a circular enactment within digitally immersive environments are only examples of where practiceled research and creative making can go. Here, the fundamental concepts and building blocks of the Santiago school are presented and reviewed in relation to their ability to inform the understanding of the nature of human experience in real and immersive worlds, and how we ought to design for it. Examples from research and practical work will help to portray how the epistemology of the Santiago school can become of interest and of real value to artistic and design practice and inquiry. Finally, the philosophical rationale guiding the inclusion of principles and concepts coming from the Santiago school in digital learning design, creative design and artistic practice not only invites us to reconsider and re-conceptualise the role of learners and of digital technology systems and tools in educational practice, but also to rethink the nature of learning and of human experience within creative practice. 